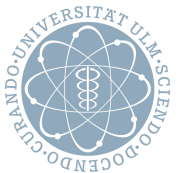

\title{
Ghost Target Identification by Analysis of the Doppler Distribution in Automotive Scenarios
}

Fabian Roos, Mohammadreza Sadeghi, Jonathan Bechter, Nils Appenrodt, Jürgen Dickmann, and Christian Waldschmidt

(C) 2017 IEEE. Personal use of this material is permitted. Permission from IEEE must be obtained for all other uses, in any current or future media, including reprinting/republishing this material for advertising or promotional purposes, creating new collective works, for resale or redistribution to servers or lists, or reuse of any copyrighted component of this work in other works.

DOI: $10.23919 /$ IRS.2017.8008128 


\title{
Ghost Target Identification by Analysis of the Doppler Distribution in Automotive Scenarios
}

\author{
Fabian Roos*, Mohammadreza Sadeghi*, Jonathan Bechter*, \\ Nils Appenrodt**, Jürgen Dickmann**, and Christian Waldschmidt* \\ *Institute of Microwave Engineering \\ Ulm University, 89081 Ulm, Germany \\ email: fabian.roos@uni-ulm.de \\ ${ }^{* *}$ Group Research and Advanced Engineering \\ Daimler AG, 89081 Ulm, Germany
}

\begin{abstract}
In an automotive environment the presence of reflecting surfaces cannot be avoided. The electromagnetic wave returning from a target vehicle can get reflected on those surfaces causing a non existing so-called ghost target. For driver assistance systems ghost targets can lead to false decisions and, therefore, they should be detected and avoided. In this paper a model for describing those ghost targets and a procedure to distinguish them from real targets using the orientation and the motion state of a vehicle is presented.
\end{abstract}

\section{Introduction}

To operate driver assistance systems safely the target parameters, i.e., orientation and dimension of a target vehicle, must be clearly identified and characterised. Reflections from a real target at reflecting surfaces as parked cars, metallic road barriers on a highway, or even larger street signs can lead to false decisions. Such unwanted targets are referred to as ghost targets and should be avoided.

The identification of ghost targets, for example in the application of through-the-wall imaging, is a well-known problem. With the precise knowledge of the position and orientation of the reflecting surface the ghost targets can be distinguished from the genuine targets as shown in [1]. A transmit beamforming can be applied as in [2] to illuminate only a certain angular domain while receiving on the whole angular domain. Thereby a mismatch between the angle of the illuminated area and the angle under which a target is located hints at a ghost target.

For the automotive case the reflecting surfaces are generally not known and, therefore, those procedures are not feasible. However, with the availability of high-resolution radar sensors extensive information about the detected targets are present, e.g., the orientation of the target vehicle can be estimated precisely as shown in [3]. In addition the measured Doppler velocity can be exploited. An example of a moving target which is detected by a next generation radar sensor is shown in Fig. 1. The radar is operating around a centre frequency of $76.45 \mathrm{GHz}$ using a bandwidth of $2 \mathrm{GHz}$ and a chirp-sequence modulation with 128 frequency ramps. With 


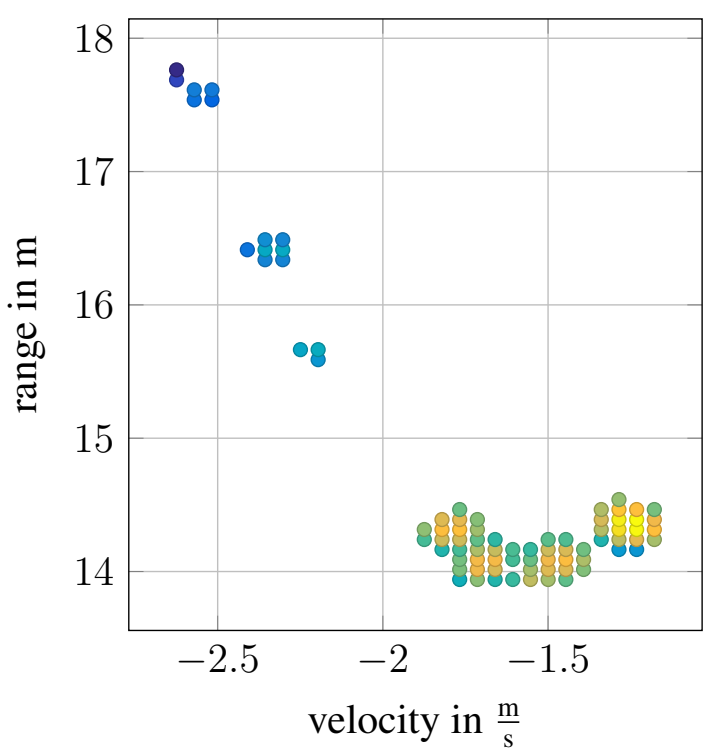

(a) The Doppler distribution of a moving target vehicle in the range-velocity plot.

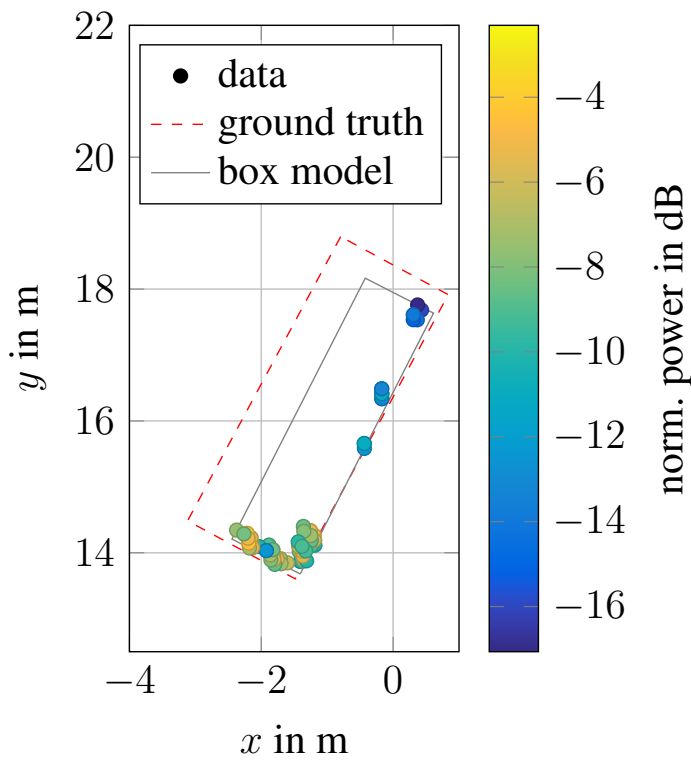

(b) The detections of the moving target lie directly on the contour $\left(--_{-}\right)$of the vehicle enabling a precise orientation estimation (- $)$.

Figure 1: A measurement of a moving target vehicle showing the Doppler distribution in (a) and the detections in (b). The colour bar is valid for both figures.

this the velocity resolution is $5.4 \frac{\mathrm{cm}}{\mathrm{s}}$. As can be seen in Fig. 1 (a) the Doppler distribution can be exploited to characterise the target. The radar reflections in (b) allow a precise orientation estimation (-).

As shown in Section 3 later on, using the Doppler distribution of the different scattering centres enables the estimation of the current motion state of the target vehicle. This determined motion state should be consistent with the current orientation of the vehicle. For a ghost target the motion state and the orientation do not match as derived in Section 2. This mismatch can be used as a criterion to identify a possible ghost target.

\section{Problem Formulation: Ghost Target Modelling}

The electromagnetic waves are reflected at different points of a target and thus multipath propagation is possible. As a positive aspect of this effect, reflections from the underbody of a vehicle can be detected and used to get a reliable model fit. However, parked vehicles at the roadside or large road signs can reflect the wave as well. The real target can then be detected under a completely different angle leading to unwanted detections.

To derive the parameters of the ghost target, the real target is assumed to be at position $P$ and a perfect electric wall, later referred to as wall, is present at a distance $a$ on the right-hand side of the sensor as depicted in Fig. 2. A mirrored sensor $S^{\prime}$ is assumed to simplify the derivation of the geometric parameters in (b). 


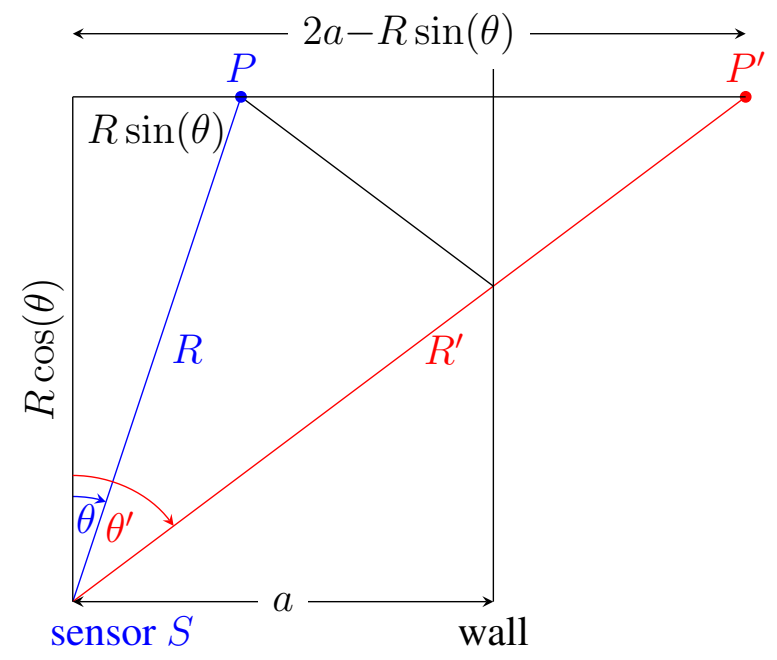

(a) Determination of the radial distance and angle under (b) which the ghost target ist detected.

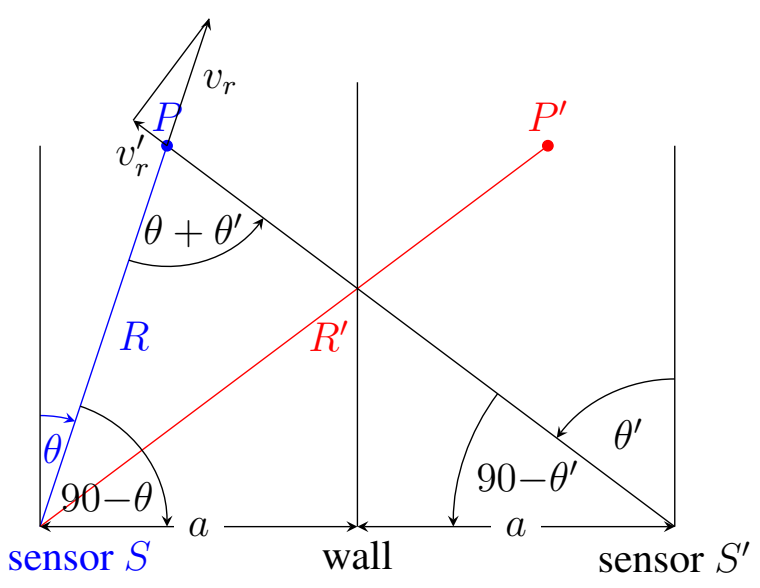

Figure 2: Geometrical model of the ghost target and derivation of the parameters range, angle, and velocity.

The distance to the ghost target $P^{\prime}$ is

$$
R^{\prime}=\sqrt{R^{2}+4 a^{2}-4 a R \sin (\theta)}
$$

while the angle is

$$
\theta^{\prime}=\arctan \left(\frac{2 a-R \sin (\theta)}{R \cos (\theta)}\right)
$$

and the component of the velocity pointing to the reflecting surface is

$$
v_{\mathrm{r}}^{\prime}=v_{\mathrm{r}} \cos \left(\theta+\theta^{\prime}\right),
$$

with $v_{\mathrm{r}}$ being the radial component of the velocity of $P$. Note that the angles are defined with respect to the ordinate.

Three ray paths are possible. First, from the sensor $S$ to the target and backwards over the wall. Second, from the sensor to the wall to the target and on the direct path backwards. Third, from the sensor to the wall to the target and the same way back. Which ray path occurs has an influence on the detected distance, angle, and velocity.

Assuming the first case, the measured distance is $R_{\mathrm{GT}}=\frac{1}{2}\left(R+R^{\prime}\right)$ and the angle is $\theta_{\mathrm{GT}}=\theta^{\prime}$. As stated in [4] the Doppler effect has an influence on the incident and reflected wave. Once as the transmitted wave hits the target and once again when it is scattered backwards. For the real target this results in a Doppler shift of

$$
f_{\mathrm{D}, \mathrm{RT}}=-\frac{f_{\mathrm{c}}}{c} 2 \vec{v} \cdot \vec{e}_{\mathrm{SP}},
$$


where $\vec{v}$ is the velocity vector of the moving target, $c$ the speed of light, $f_{\mathrm{c}}$ the transmitted centre frequency, and $\vec{e}_{\mathrm{SP}}$ the unit vector from the sensor to the target $P$. For the ghost target the Doppler shift

$$
f_{\mathrm{D}, \mathrm{GT}}=f_{\mathrm{c}}\left(\frac{-\vec{v} \cdot \vec{e}_{\mathrm{S}^{\prime} \mathrm{P}}-\vec{v} \cdot \vec{e}_{\mathrm{SP}}}{c+\vec{v} \cdot \vec{e}_{\mathrm{S}^{\prime} \mathrm{P}}}\right) \approx-\frac{f_{\mathrm{c}}}{c}\left(\vec{v} \cdot \vec{e}_{\mathrm{S}^{\prime} \mathrm{P}}+\vec{v} \cdot \vec{e}_{\mathrm{SP}}\right)
$$

results in a combination of the velocity in radial direction and in the direction to the wall, where $\vec{e}_{S^{\prime} \mathrm{P}}$ is the unit vector from the mirrored sensor position to the target. This frequency shift resulting from the scattering of a moving object is also used to measure the velocity of fluids in laser Doppler velocimetry.

In the second case the ghost target is detected behind the real target so only the angle of the ghost target changes while the other parameters are unchanged. For the third case the distance increases because the wave gets reflected on the wall twice, therefore, only the angle is equal. For simplicity and a better graphic representation only the first case is shown in the following.

\section{Motion Estimation}

A high-resolution radar sensor enables the possibility to detect several different scattering centres on a target vehicle as shown in Fig. 1. A vehicle can be modelled as presented in [5] by a set of different scattering centres. Assuming a linear motion, each point and therefore each scattering centre on the target has the same velocity. This is shown in Fig. 3 (a) where each scattering centre $(\circ)$ has the same velocity vector $(\longrightarrow)$. Depending on the angle under which the target is visible, the radial velocity $(\longrightarrow)$ differs.

If the vehicle is turning, i.e., a yaw rate $\omega$ is present, for each point $Q$ on the vehicle the velocity is altered by

$$
\begin{aligned}
\vec{v}_{Q} & =\vec{v}_{\mathrm{A}}+\vec{\omega} \times \vec{d}_{\mathrm{A} Q} \\
\left(\begin{array}{c}
v_{Q}^{x} \\
v_{Q}^{y} \\
0
\end{array}\right) & =\left(\begin{array}{c}
v_{\mathrm{A}}^{x} \\
v_{\mathrm{A}}^{y} \\
0
\end{array}\right)+\left(\begin{array}{c}
0 \\
0 \\
\omega
\end{array}\right) \times\left(\begin{array}{c}
x_{Q}-x_{\mathrm{A}} \\
y_{Q}-y_{\mathrm{A}} \\
0
\end{array}\right)
\end{aligned}
$$

except for the rotation centre which is the middle point of the rear axle A. The vector from the rotation centre to the point $Q$ is written as $\vec{d}_{\mathrm{A} Q}$ and the movement vector in the rotation centre is given by $\vec{v}_{\mathrm{A}}$. The velocity in $x$ direction in the point $Q$ ist denoted by $v_{Q}^{x}$. For a yaw rate $\omega=-1 \frac{\mathrm{rad}}{\mathrm{s}}$ the resulting velocities are shown in Fig. 3 (b).

The complete motion state of a vehicle can be described as in [6] with the motion vector

$$
\vec{m}_{Q}=\left(\begin{array}{c}
\omega \\
v_{Q}^{x} \\
v_{Q}^{y}
\end{array}\right)
$$




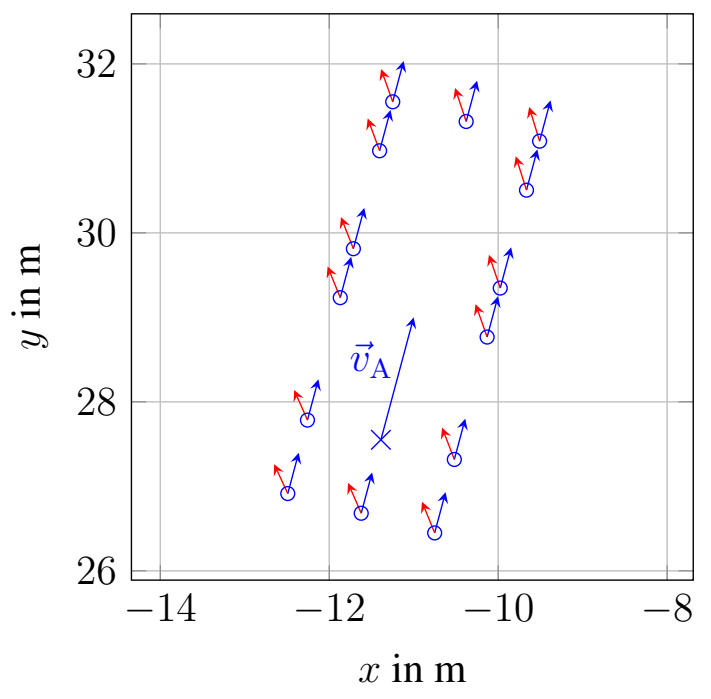

(a) A linear motion with $\omega=0 \frac{\mathrm{rad}}{\mathrm{s}}$.

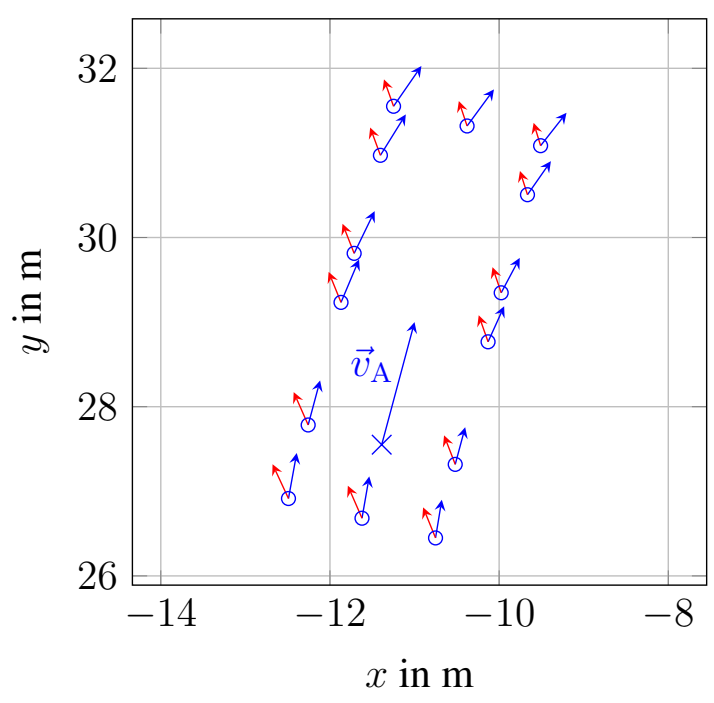

(b) A turning motion with $\omega=-1 \frac{\mathrm{rad}}{\mathrm{s}}$.

Figure 3: Example of a vehicle which moves with a constant velocity indicated by the large movement vector in the middle. For each assumed scattering centre $(\circ)$ the velocity vector $(\longrightarrow)$ is shown with respect to the yaw rate $\omega$ and the rotation centre, which is the rear axle $(X)$. For a sensor positioned in the point of origin the radial part $(\longrightarrow)$ of the velocity can be observed.

in an arbitrary point $Q$.

As there are three degrees of freedom in the motion vector using a single radar sensor is not sufficient as shown in [6] to estimate the complete motion state. Therefore two radar sensors at different positions enable the estimation in a single measurement. This is done with the equation system

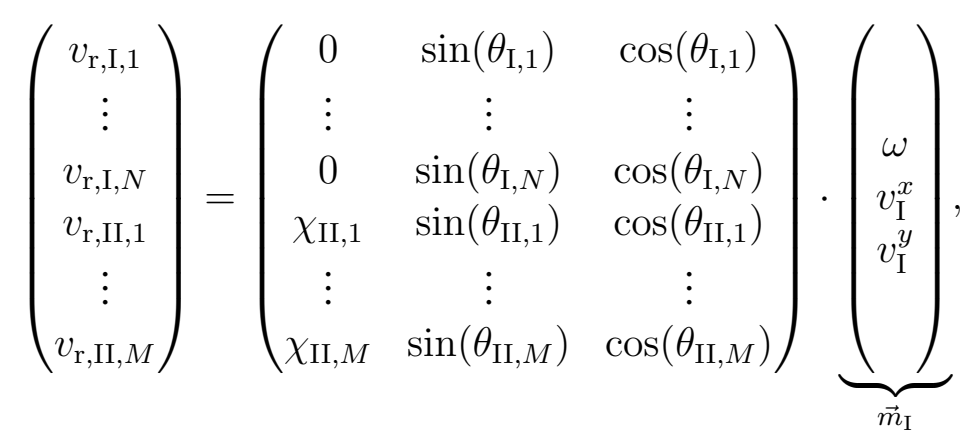

with $v_{\mathrm{r}, \mathrm{I}, j} j \in\{1, \ldots, N\}$ being the measured Doppler velocities of the first sensor and $v_{\mathrm{r}, \mathrm{II}, k} k \in$ $\{1, \ldots, M\}$ the Doppler velocities of the second sensor. The angle to the first target of the first sensor is $\theta_{\mathrm{I}, 1}$ with respect to the ordinate. The origin of the used coordinate system is in the first sensor position, thus the expression $\chi_{\mathrm{II}, k}=-y_{\mathrm{II}} \sin \left(\theta_{\mathrm{II}, k}\right)+x_{\mathrm{II}} \cos \left(\theta_{\mathrm{II}, k}\right)$ considers the offset $\left(x_{\mathrm{II}}, y_{\mathrm{II}}\right)$ to the second sensor.

The estimated motion vector $\vec{m}_{\mathrm{I}}$ represents the motion state at the representation point in the origin of the coordinate system. To be able to compare it later in Section 4 with the estimated orientation, it needs to be transformed into the rotation centre of the target vehicle. This can be 
done with the transformation matrix

$$
\left(\begin{array}{c}
\omega \\
v_{\mathrm{A}}^{x} \\
v_{\mathrm{A}}^{y}
\end{array}\right)=\left(\begin{array}{ccc}
1 & 0 & 0 \\
-y_{\mathrm{A}} & 1 & 0 \\
x_{\mathrm{A}} & 0 & 1
\end{array}\right) \cdot\left(\begin{array}{c}
\omega \\
v_{\mathrm{I}}^{x} \\
v_{\mathrm{I}}^{y}
\end{array}\right) .
$$

The vector $\left(v_{\mathrm{A}}^{x}, \quad v_{\mathrm{A}}^{y}\right)^{\mathrm{T}}$ describes the current movement of the target, and the angle of this vector is later referred to as the angle of the motion vector.

\section{Ghost Target Identification: Simulation Results}

The frequency shift of the scattering of a moving object in combination with the reflection on a surface (5) leads to a motion vector (10) which does not fit to the orientation of the vehicle. This misalignment can be used to distinguish a real target from a ghost target. The orientation of a target can be estimated with a tracking of the target over time. If a high-resolution radar sensor is used, the reflections may allow an orientation estimation as presented in [3]. This means that a single measurement is sufficient to decide if a detected target is a real or a ghost target.

To validate the ghost target identification, a chirp-sequence radar is simulated with the parameters listed in Tab. 1. As a uniform linear array is assumed the angle estimation is done using a Fourier transform. A target vehicle is placed at $(-10 \mathrm{~m}, 29 \mathrm{~m})$ with an angle of $75^{\circ}$ and the scattering centres are assumed as in Fig. 3. The wall is located at $x=5 \mathrm{~m}$ parallel to the boresight.

For a linear motion the simulation results are shown in Fig. 4. In the $x-y$ plot in (a), the left target is reflected at the wall (-) and visible right of it. In the range-velocity plot in (b), the ghost target is detected with closer range but appears as a valid target. In this domain the orderedstatistic constant false alarm rate (OS-CFAR) algorithm described in [7] is applied to distinguish targets from noise. Afterwards a simple peak search is applied to extract the scattering centres and for segmentation the density based spatial clustering of applications with noise (DBSCAN) algorithm introduced in [8] is used to remove possible noise and outlier points not fitting to the

Table 1: Specifications of Chirp-Sequence Radar Simulations

\begin{tabular}{lr} 
Parameter & Value \\
\hline carrier frequency $f_{\mathrm{c}}$ & $77 \mathrm{GHz}$ \\
bandwidth $B$ & $1 \mathrm{GHz}$ \\
chirp duration $T_{\mathrm{c}}$ & $20 \mu \mathrm{s}$ \\
chirp repetition time $T_{\mathrm{r}}$ & $25 \mu \mathrm{s}$ \\
sampling frequency $f_{\mathrm{s}}$ & $30 \mathrm{MHz}$ \\
number of chirps $L$ & 512 \\
number of receiving elements & 20 \\
element distance in $\lambda$ & 0.545 \\
window function & Hann window \\
zero padding & signal length doubled
\end{tabular}




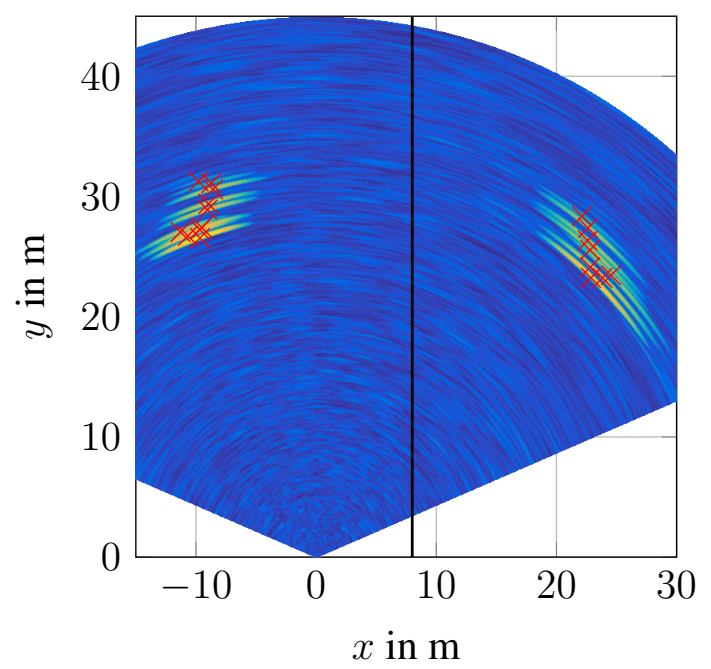

(a) The left target gets reflected at the wall (-).

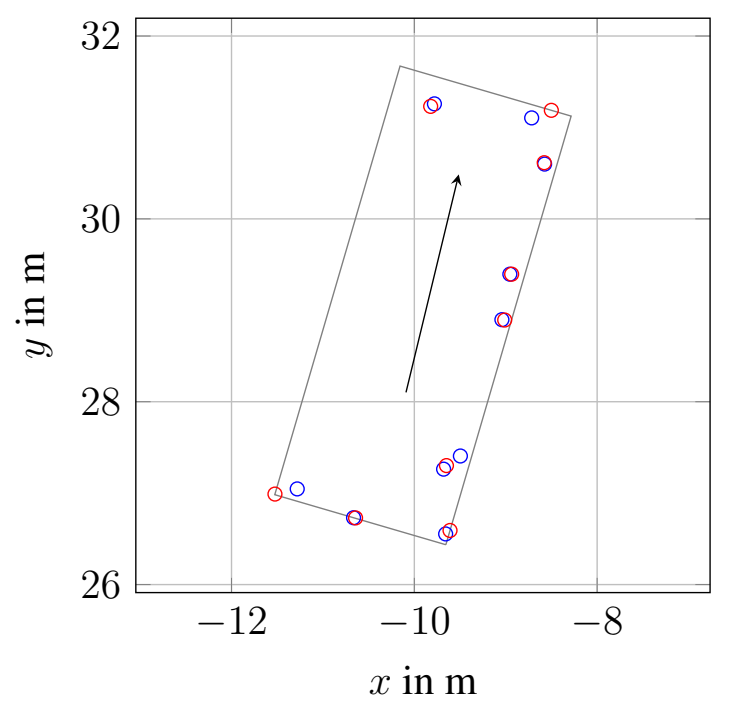

(c) Extracted peaks of the real target from the left $(\circ)$ and right $(\circ)$ sensor. With a box model $(-)$ the orientation is estimated to $74^{\circ}$ and the motion vector $\left(\longrightarrow\right.$ ) indicates an orientation of $76^{\circ}$.

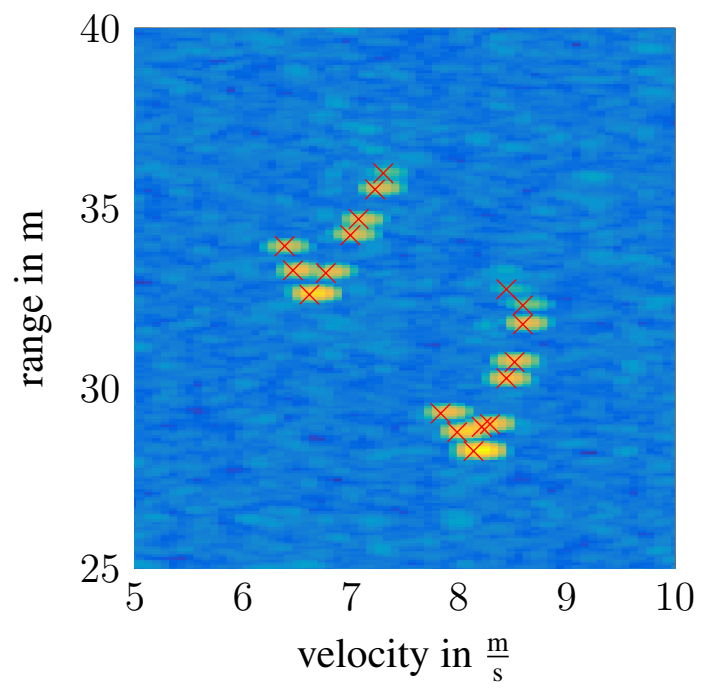

(b) Range-velocity plot of the real and ghost target.

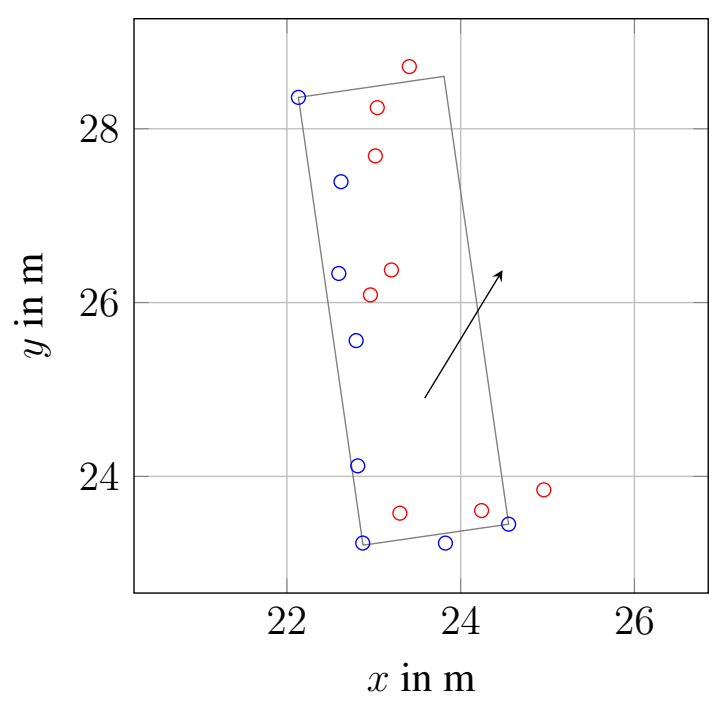

(d) Extracted peaks of the ghost target from the left ( $($ ) and right ( $($ ) sensor. With a box model ( - ) the orienation is estimated to $98^{\circ}$ and the motion vector $\left(\longrightarrow\right.$ ) indicates an orientation of $59^{\circ}$.

Figure 4: Simulation of a chirp-sequence radar with a target in linear motion placed left of a reflecting wall. The $x-y$ plot of the scene is shown in (a), while the range-velocity plot is shown in (b). After a peak detection the orientation and the motion vector are estimated of the real target in (c) and of the ghost target in (d).

current model. This is done for the data of the left (o) and right (o) sensor and shown for the real target in (c). A box model algorithm from [3] is applied to the clustered points of both sensors to estimate the orientation $(-)$ which is then compared to the motion vector $(\longrightarrow)$.

In the case of the real target, the orientation estimation result of $74^{\circ}$ yields nearly the same angle as the motion vector $76^{\circ}$, but for the ghost target in (d) the two angles, $98^{\circ}$ for the orientation 
and $59^{\circ}$ for the motion vector, differ considerably. This discrepancy is used for classification.

In Fig. 5 the vehicle is turning with a yaw rate of $\omega=-0.5 \frac{\mathrm{rad}}{\mathrm{s}}$. Once again for the real target in (c) the orientation of $70^{\circ}$ is in accordance with the motion vector angle of $73^{\circ}$, while for the ghost target in (d) the discrepancy with $93^{\circ}$ respectively $25^{\circ}$ is again present.

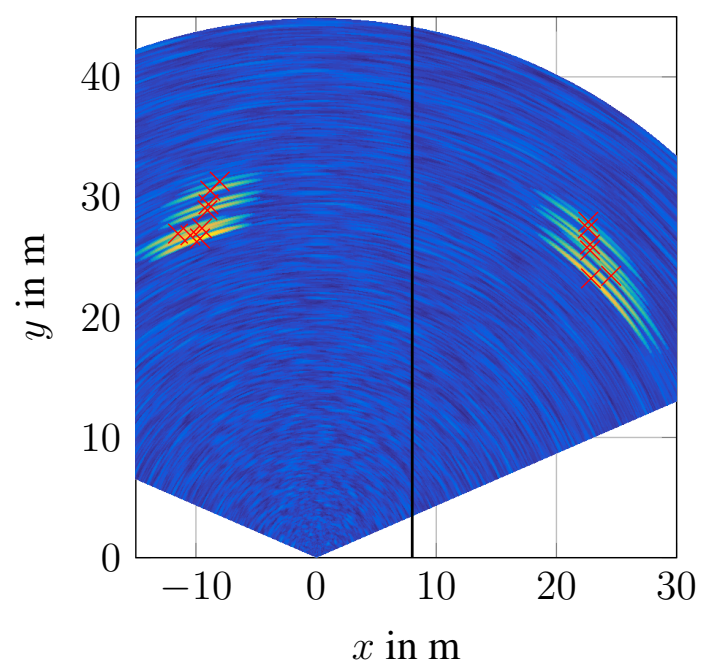

(a) The left target gets reflected at the wall (-).

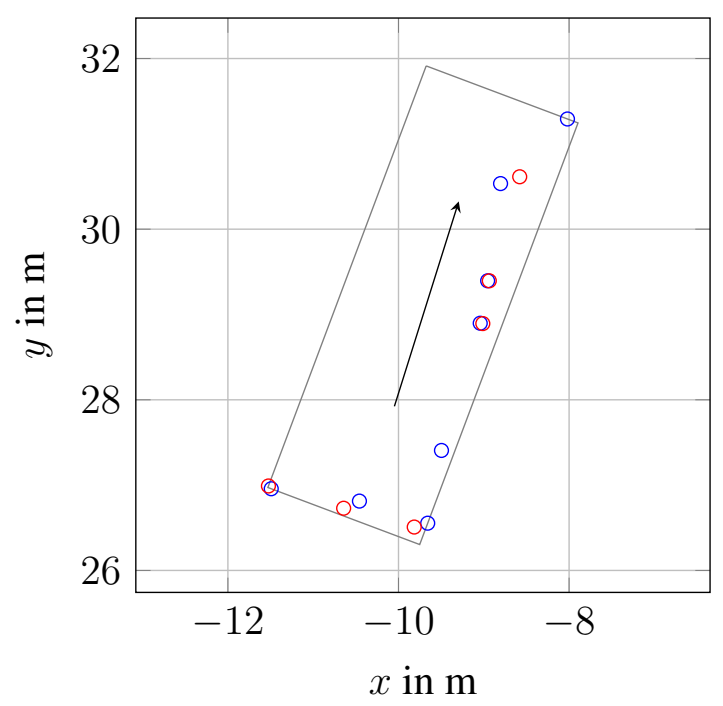

(c) Extracted peaks of the real target from the left $(\circ)$ and right $(\circ)$ sensor. With a box model $(-)$ the orientation is estimated to $70^{\circ}$ and the motion vector $(\longrightarrow)$ indicates an orientation of $73^{\circ}$.

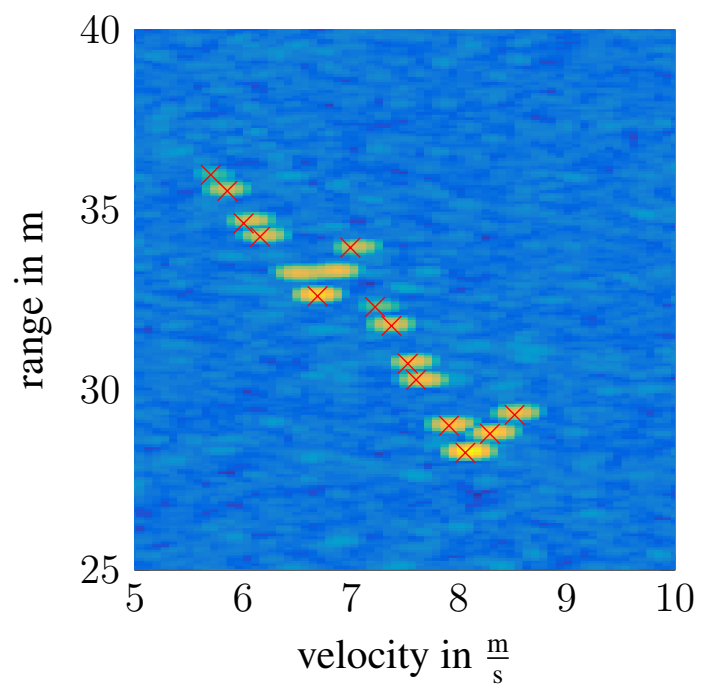

(b) Range-velocity plot of the real and ghost target.

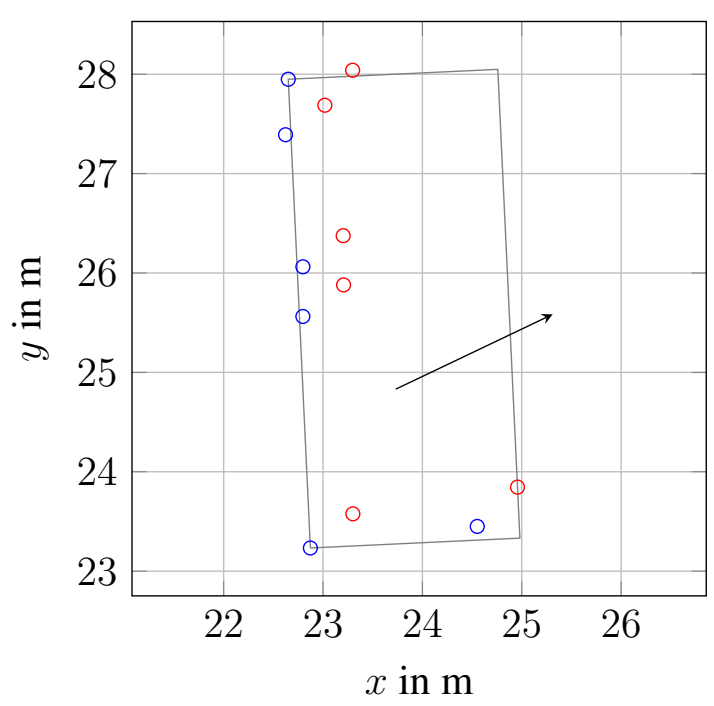

(d) Extracted peaks of the ghost target from the left (०) and right ( $($ ) sensor. With a box model ( - ) the orienation is estimated to $93^{\circ}$ and the motion vector $(\longrightarrow)$ indicates an orientation of $25^{\circ}$.

Figure 5: Simulation of a chirp-sequence radar with a target with a yaw rate of $\omega=-0.5 \frac{\mathrm{rad}}{\mathrm{s}}$ placed left of a reflecting wall. The $x-y$ plot of the scene is shown in (a), while the range-velocity plot is shown in (b). After a peak detection the orientation and the motion vector are estimated of the real target in (c) and of the ghost target in (d). 


\section{Conclusion}

Due to reflections on surfaces ghost targets can occur. A model is presented to describe the parameters of ghost targets. From the extracted scattering centres the orientation is estimated using a box model and compared to the angle of the motion vector. If those angles are nearly equal this indicates a real target, while for a ghost target a large deviation is present. Even for a turning vehicle where a yaw rate is present, the presented approach can be used. However, in this case two radar sensors are required to correctly estimate the motion vector.

\section{References}

[1] L. Qiu, T. Jin, and Z. Zhou, "Multipath model and ghosts localization in ultra-wide band virtual aperture radar," in 12th International Conference on Signal Processing (ICSP), Oct. 2014, pp. 2149 2152 .

[2] I. Vermesan, D. Carsenat, C. Decroze, and S. Reynaud, "Ghost image cancellation algorithm through numeric beamforming for multi-antenna radar imaging," IET Radar, Sonar and Navigation, vol. 7, no. 5, pp. 480-488, Jun. 2013.

[3] F. Roos, D. Kellner, J. Dickmann, and C. Waldschmidt, "Reliable Orientation Estimation of Vehicles in High-Resolution Radar Images," IEEE Transactions on Microwave Theory and Techniques, vol. 64, no. 9, pp. 2986-2993, Sep. 2016.

[4] P. A. Tipler and G. Mosca, Physik für Wissenschaftler und Ingenieure, 2nd ed., D. Pelte, Ed. Spektrum Akademischer Verlag, 2007.

[5] M. Bühren and B. Yang, "Automotive Radar Target List Simulation based on Reflection Center Representation of Objects," in Workshop on Intelligent Transportation (WIT), Hamburg, Germany, Mar. 2006, pp. 161-166.

[6] D. Kellner, M. Barjenbruch, J. Klappstein, J. Dickmann, and K. Dietmayer, "Tracking of Extended Objects with High-Resolution Doppler Radar,' IEEE Transactions on Intelligent Transportation Systems, vol. 17, no. 5, pp. 1341-1353, Dec. 2016.

[7] H. Rohling, "Radar CFAR Thresholding in Clutter and Multiple Target Situations," IEEE Transactions on Aerospace and Electronic Systems, vol. AES-19, no. 4, pp. 608-621, Jul. 1983.

[8] M. Ester, H.-P. Kriegel, J. Sander, and X. Xu, "A Density-Based Algorithm for Discovering Clusters in Large Spatial Databases with Noise," in Proceedings of 2nd International Conference on Knowledge Discovery and Data Mining. AAAI Press, 1996, pp. 226-231. 